\title{
Mechanism of Elastic Waves Reflection in Geological Media
}

\author{
Viktor Sidorov, Michail Tarantin \\ Organization of Russian Academy of Sciences, Mining Institute Ural Branch RAS, Perm, Russia \\ Email: gp_svk@mi-perm.ru
}

Received October 20, 2011; revised November 22, 2011; accepted December 14, 2011

\begin{abstract}
Reflecting properties of layered geological media are substantiated in the framework of phonon-phonon mechanism of elastic wave propagation in porous media. In this scope the reflection coefficient is calculated using not impedances but impulses of phonons in adjoining porous media. Assuming for the first approximation that rocks do fulfill an average time equation we got an expression for the reflection coefficient via porosity factors of that geological medium. For calculation of reflection coefficient the wavelength is chosen as averaging line scale. These coefficients are calculated at every depth point for a set of frequencies in seismic range. Resulting curves have special depth points. Being crossplotted in time-frequency space such points do form coherent units. These units we call effective boundaries, because they cause all reflections for the given media in the framework of considered model. Effective boundaries are not wide-band as for two half spaces but have a cutoff at some low frequency. Geological medium at a whole is characterized by the system of such effective boundaries that are capable to form a reflection waves field. To construct this field an algorithm is developed that solves the direct problem of seismic in the framework of effective boundaries theory. This algorithm is illustrated with vibroseis survey modeling for a specific geological section.
\end{abstract}

Keywords: Elastic Waves; Phonons; Heterogeneous Media; Reflection Coefficient; Direct Seismic Problem; Effective Seismic Boundaries

\section{Introduction}

We proposed a mechanism of elastic waves propagation in rocks [1] that justified an independence of logarithmic decrement on frequency or, equivalent, justified an absence of velocity dispersion when an attenuation does take place. The problem of decrement arose long time ago [2] but is still not solved and the proposed mechanism as far as we know is unique. That is why we do not consider many models of elastic waves propagation in heterogeneous media among which the Biot model $[3,4]$ is the most popular. This model describes the elastic waves propagation in the media with the solid matrix and many recent works attend to it [5-7]. But this model does not comply with the frequency independence of decrement.

Proposed mechanism supposes the elastic wave propagation in heterogeneous media to be the process that is attended and supported by phonon-phonon interactions of a special sort. At that the correspondence is proved of some phonon impulses and their combinations with elastic wave parameters. Justification is based on a specific type of heterogeneous medium-porous fluid-saturated rocks with an average time equation fulfilled. These were the media where an experimental testing of a proposed mechanism was allowed. For that purposes laboratory data and acoustic logging material in carbonate forma- tions were analyzed $[1,8]$.

The aim of the paper is to substantiate the reflection properties of layered geological media in the framework of phonon-phonon mechanism and to create on that basis an algorithm for solving the direct seismic problem.

\section{The Reflection Coefficient Derivation}

For a special type of porous fluid-saturated media the longitudinal wave propagation is attended and supported by phonon interactions that are described by an impulse conservation law expression:

$$
\frac{h v}{V_{P m}}-\frac{m h v}{V_{P m}}+\frac{m h v}{V_{f l}}=\frac{h v}{V_{P}}
$$

In the expression above $m$ denotes the porosity coefficient, $V_{P m}$-velocity of longitudinal waves in solid matrix, $V_{f l}$-velocity in the fluid, $V_{p}$-velocity in the porous medium, $h$ is the Plank constant and $v$ is the phonon frequency. It is stated in mechanism that phonon's velocities are equal to that of waves in the corresponding components of the porous medium.

Phonons with impulses $m h v / V_{P m}$ and $m h v / V_{f l}$ are responsible for wave energy dissipation because they are directly proportional to porosity $m$ and frequency $v$ and correspond to attenuation coefficient in porous fluid-sa- 
turated media with the average time equation fulfilled. Such kind of media in turn has an attenuation coefficient direct proportional to wave porosity and frequency $f$ [1]: $\alpha \propto f \cdot m$.

We supposed that reflection properties of a layered geological medium could be gained in the framework of phonon-phonon mechanism. Let's consider from this point of view the reflection coefficient for two-media boundary. In the common form it could be written as

$$
R=\frac{p_{1}-p_{2}}{p_{1}+p_{2}}
$$

where $p$ is the impulse, that turns a production of density and velocity for elastic waves $(p=\sigma V)$ and is wellknown as impedance. But this time $p$ is the phonon impulse: $p=h v / V$.

Let's consider two adjoined media with porosities $m_{1}$ and $m_{2}$. Impulses in the reflection coefficient (2) we express from left part of (1) with porosities $m_{1}$ and $m_{2}$. And so we got

$$
R=\frac{\left(\frac{h v}{V_{P m}}-\frac{m_{1} h v}{V_{P m}}+\frac{m_{1} h v}{V_{f l}}\right)-\left(\frac{h v}{V_{P m}}-\frac{m_{2} h v}{V_{P m}}+\frac{m_{2} h v}{V_{f l}}\right)}{\left(\frac{h v}{V_{P m}}-\frac{m_{1} h v}{V_{P m}}+\frac{m_{1} h v}{V_{f l}}\right)+\left(\frac{h v}{V_{P m}}-\frac{m_{2} h v}{V_{P m}}+\frac{m_{2} h v}{V_{f l}}\right)}
$$

Simple transformations lead us to:

$$
R=\frac{\Delta m\left(V_{f l}^{-1}-V_{P m}^{-1}\right)}{2 V^{-1}}
$$

where $\Delta m=m_{1}-m_{2}$ and $V^{-1}$ is the average interval time for two media.

So we have an expression for reflection coefficient for
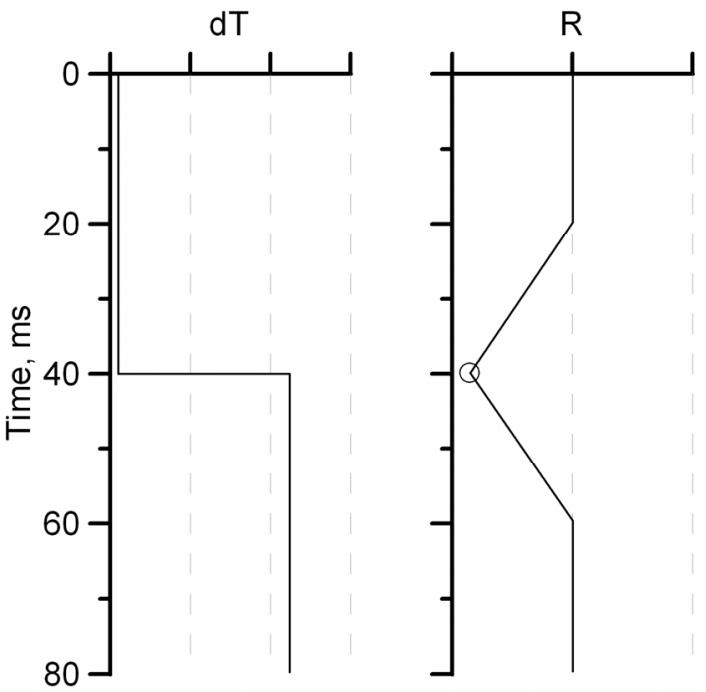

(a) a boundary of two porous media based on the expression for phonon interaction. It should be marked that exactly the same result could be gained if the reflection coefficient (2) would be expressed via impulses $p=\sigma V$ when neglecting density changes and assuming that in adjoined media velocities are calculated using the average time equation. But such a definition would be tautological because for porous media the average interval time expression used in this approach is the consequence of the phonon impulse conservation law.

The next step is to use the coefficient $R$ to determine the reflection properties of a layered geological media. We suppose from the beginning that rocks could be classified as reference medium-porous fluid-saturated with average time equation fulfilled. Let's calculate the expression (4) for a moving window along the medium cross-section. At the boundary of two spaces this function will have an extremum corresponded to the real reflection coefficient for that media boundary (Figure 1(a)). For a single layer calculated function has a shape similar to that shown in Figure 1(b). Two kink points mark the layer boundaries and the corresponding reflection coefficients. As a size of moving window it is naturally to take a wavelength because the phonon-phonon interaction is corresponded to the specific elastic wave frequency. And then an algorithm of calculations would be as follows.

The log-curve of an interval time (reversal velocity) is transformed from depth-scale into time-scale. The value of frequency $f_{n}$ is set. Along the geological section with a special step moves the interval of a wavelength size with the reference point at the center. Above and below the reference point the function (4) is calculated with $m$ being equal to difference of porosity factors for upper and
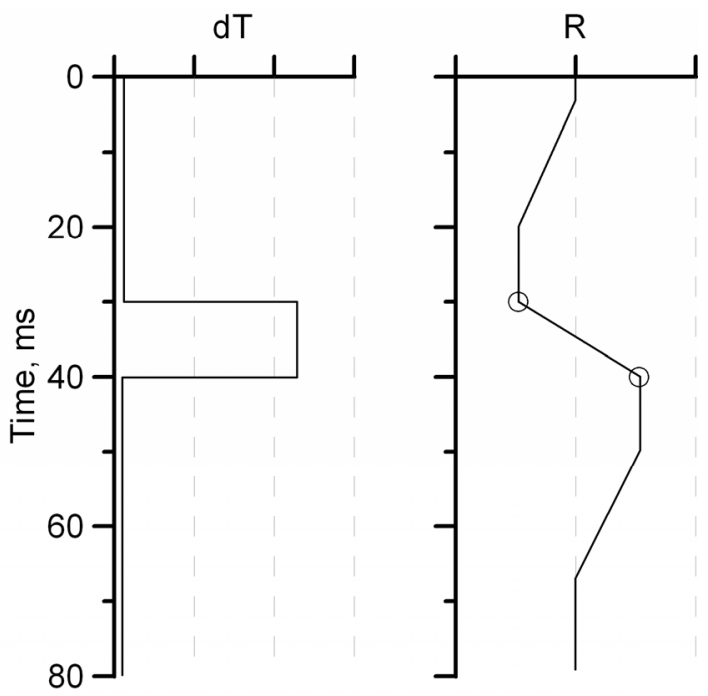

(b)

Figure 1. The view of reflection coefficient curves near two half-space boundary (a) and near thin layer (b); dT: interval time of wave, $R$ : reflection coefficient. 
lower half-interval and $V^{-1}$ —an average interval time for all wavelength interval. Such a way the reflection function $R\left(f_{n}, t\right)$ is calculated. For a general randomly layered geological medium the corresponding curve have extreme-points and kink-points. Such curves are calculated for a count of frequencies within a selected seismic range. For every curve all special points are registered: frequencies, times and coefficient values. Being plotted in the $\left(f_{n} ; t\right)$ plane these points make separate boundaries. Let's call these boundaries effective ones. These boundaries accumulate seismic reflections of a layered stratum of rocks for a selected frequency band and this fact clears a distinctly new approach to reflected waves field calculation.

\section{Direct Seismic Problem Solving Using Effective Boundaries Approach}

Let's demonstrate the process of signal calculation using effective boundaries approach with the specific example of vibroseis method. Geological section is presented by carbonate rocks with an embedded unit of terrigenous sediments (Figure 2). Vertical scale is time in ms and the start time is taken by convention. Information about velocity is given as log curve of longitudinal waves inverse velocities. Using this curve and assuming that considering rocks do fulfill an average time equation parameters for $R\left(f_{n}, t\right)$ calculation are determined. These functions are calculated for series $f_{n}$ of frequencies within seismic band, and one for $f=50 \mathrm{~Hz}$ is presented as an example in the corresponding column (see Figure 2).
All curves $R\left(f_{n}, t\right)$ are examined for extremes and kinks. These points being plotted in frequency-time coordinates with mark-size depending on the reflection coefficient value make coherent units as can be seen in the next column in Figure 2. These coherent units were defined above as effective boundaries. Such kind of boundaries in general are not wide-band like in the case of two half spaces but are limited in frequency especially in low-frequency range. Every boundary has its own frequency dependence of reflection.

Representation of cross-section model as a chain of effective boundaries allows the calculation of reflections from the given geological section to be simply enough: the result would be the composition of reflections from every one of effective boundaries. Due to the special properties of these new objects-variations of reflection coefficient with depth (or time) and frequency-it is convenient to calculate the reflection function not for every boundary but for the section at once. For that purpose for every frequency $f_{n}$ within the selected seismic band the geometrical sum of all reflected signals allowed for that frequency is calculated. The differences in the registration times of reflected wavelets are accounted in the phase part of the complex coefficient while the real part is responsible for the amplitude of signal regarding its sign. As a result, the medium under consideration is characterized by the spectral function:

$$
R\left(f_{n}\right)=\sum_{i} a_{i} \exp \left(j \phi_{i}\right)
$$

where $i$ accounts for all effective boundaries of the stratum at the frequency $f_{n}$.

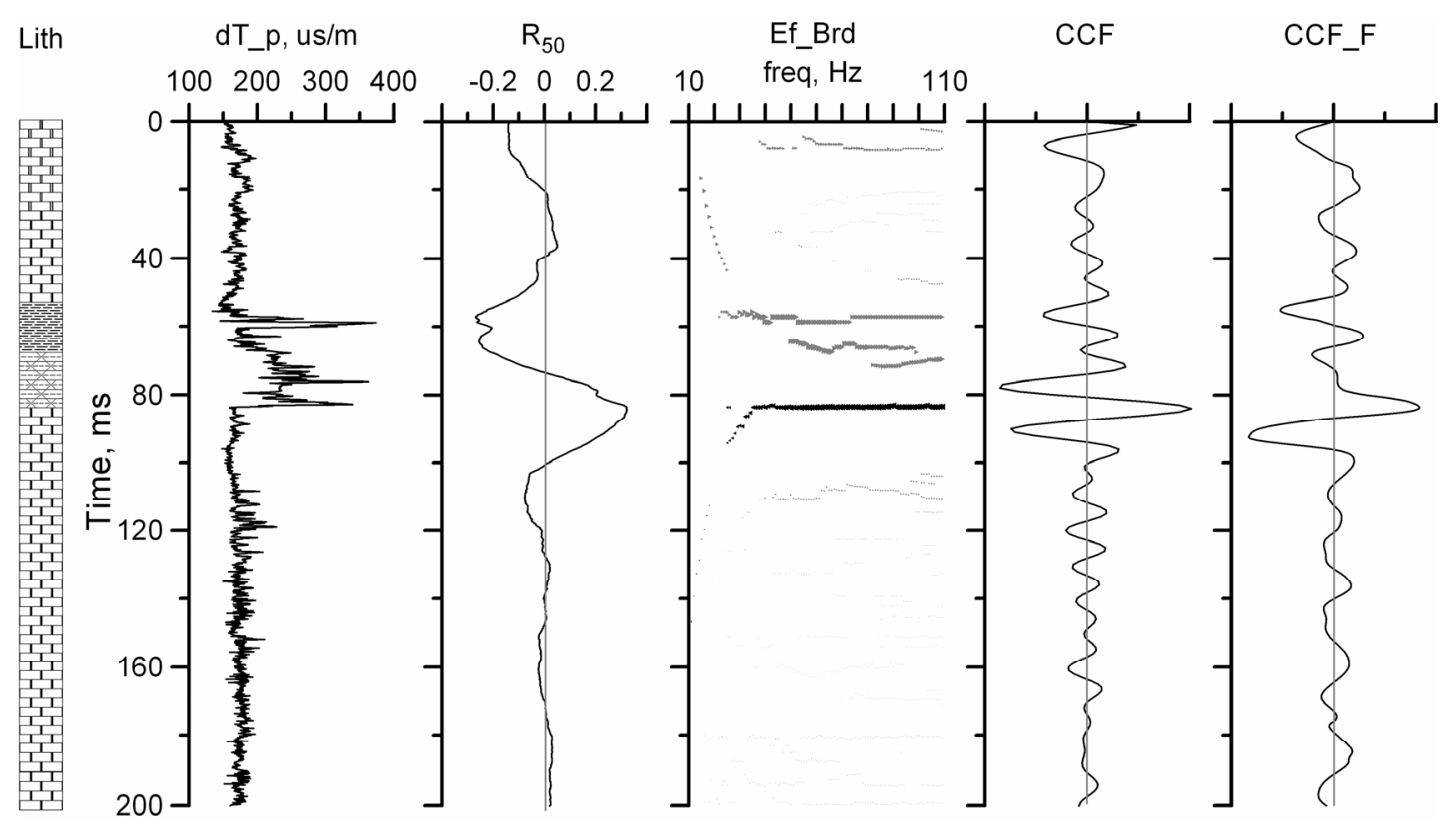

Figure 2. Calculation of cross-correlation function (CCF) of reflected signal for vibroseis method; Lith: lithological section, dT_p: inverse velocity of longitudinal waves, $R_{50}$ : reflection coefficient for a frequency $50 \mathrm{~Hz}$, Ef_Brd: selected extremes of reflection function, CCF: cross-correlation function of source sweep and reflected signal, CCF_F: processed field seismic trace. 
This function accumulates reflection properties of all effective boundaries. The reflected signal could be gained by convolution of this function and a signal accepted for a radiated one. If the radiated signal is sweep then the result of convolution should be correlated to that sweep in addition.

In the example in the Figure 2 the source signal is sweep with frequencies $14-100 \mathrm{~Hz}$. The result of calculations using presented algorithm is shown in the 5-th column; the most right column demonstrate fragment of seismic trace gained in the standard processing of field data in the framework of vibroseis method. It is seen that modeled signal does not conflict with the real one and has its main features.

\section{Conclusions and Discussion}

Described mechanism of reflection arises as the consequence of phonon-phonon mechanism of elastic wave propagation in rocks. Its development gave a distinctly different from the others model for geologic stratum approximation - the model of medium with effective boundaries. Its features are clearly seen when compared with the layered medium. First of all, in the layered medium all layer borders are caused by change in velocity (or impedances) while effective boundaries have different physical nature and are caused by heterogeneity. It is heterogeneity of rocks that makes elastic wave to loose its energy by way of scattered phonons. Abrupt change of the heterogeneity parameter, or porosity factor in our case, causes the appearance of effective boundary. Second, in layered medium the value of reflection coefficient for every boundary does not depend on frequency. Dependence on frequency appears only for a system of such boundaries. Effective boundaries (its depths and coefficients) in general do depend on frequency a priori because the algorithm for their construction involves the wavelengths at the averaging stage. Third, the layered medium being sequentially applied to the system of layers gives a resonant system $[9,10]$. This fact complicates modeling so some methods of regularization are involved. For example, only primary reflections could be used and this gives us not resonant but more stable interference system [11]. The model with effective boundaries in this sense is more stable from the start.

\section{REFERENCES}

[1] V. K. Sidorov and M. V. Tarantin, "A Possible Mechanism for Elastic Wave Propagation in Porous Rocks," Doklady Earth Sciences, Vol. 434, No. 1, 2010, pp. 12531256. doi:10.1134/S1028334X10090242

[2] L. Knopoff, “Attenuation of Elastic Waves in the Earth,” In: W. P. Mason, Ed., Physical Acoustics: Principles and Methods, Academic Press, New York, 1965, pp. 287-324.

[3] M. A. Biot, "Theory of Propagation of Elastic Waves in a Fluid Saturated Porous Solid, 1. Lower Frequency Range," Journal of the Acoustical Society of America, Vol. 28, No. 2, 1956, pp. 168-178. doi:10.1121/1.1908239

[4] M. A. Biot, "Theory of Propagation of Elastic Waves in a Fluid Saturated Porous Solid, 2. Higher Frequency Range," Journal of the Acoustical Society of America, Vol. 28, 1956, pp. 178-191.

[5] J. R. Dvorkin, R. Nolen-Hoeksema and A. Nur, "The Squirt-Flow Mechanism: Macroscopic Description,” Geophysics, Vol. 59, No. 3, 1994, pp. 428-438. doi:10.1190/1.1443605

[6] G. A. Gist, "Fluid Effects on Velocity and Attenuation in Sandstones," Journal of the Acoustical Society of America, Vol. 96, No. 2, 1994, pp. 1158-1173. doi:10.1121/1.410389

[7] J. M. Carcione, C. Morency and J. E. Santos, "Computational Poroelasticity-A Review,” Geophysics, Vol. 75, No. 5, 2010, pp. 75A229-75A243.

[8] V. K. Sidorov and M. V. Tarantin, "A Method for Estimation of a Dynamic Properties of Elastic Waves in Acoustic Logs," Geophysica, Vol. 3, 2011, pp. 7-12.

[9] L. M. Brekhovskih, "Waves in Layered Media," 2nd Edition, Academic Press, New York, 1980.

[10] L. M. Brekhovskih and O. A. Godin, “Acoustics of Layered Media,” Nauka, Moscow, 1989 (in Russian).

[11] G. N. Gogonenkov, "Synthetic Seismograms Calculation and Application,” Nedra, Moscow, 1972 (in Russian). 